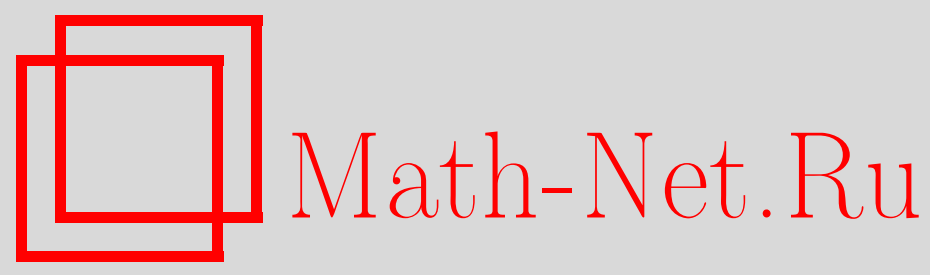

И. В. Добрынина, О ширине в свободных произведениях с объединением, Матем. заметки, 2000, том 68, выпуск $3,353-359$

DOI: https://doi.org/10.4213/mzm952

Использование Общероссийского математического портала Math-Net.Ru подразумевает, что вы прочитали и согласны с пользовательским соглашением http://www.mathnet.ru/rus/agreement

Параметры загрузки:

IP : 54.224 .60 .19

26 апреля 2023 г., 03:35:50

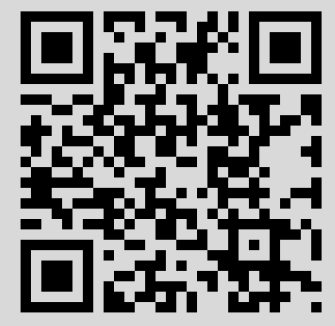




\section{О ШИРИНЕ В СВОБОДНЫХ \\ ПРОИЗВЕДЕНИЯХ С ОБЪЕДИНЕНИЕМ}

\section{И.В. Добрынина}

В данной работе рассматривается проблема конечной ширины для свободных произведений групп с объединенной подгруппой.

Библиограффия: 6 названий.

Следуя Ю.И. Мерзлякову [1], под иириной $\operatorname{wid}(G, \varphi)$ вербальной подгруппы $\varphi(G)$, определенной в группе $G$ словом $\varphi$, будем понимать наименьшее $m \in \mathbb{N} \cup\{+\infty\}$ такое, что всякий элемент подгрупшы $\varphi(G)$ записьвается в виде произведения $\leqslant m$ значений слова $\varphi$.

Слово $\varphi$ будем называть собственным, если $\varphi(G) \neq 1$ и $\varphi(G) \neq G$. Соответствующую подгрупу $\varphi(G)$ также будем назьвать собственной.

А. Х. Ремтула [2] доказал, что в нетривиальном свободном произведении $A * B$ ширина всякой собственной вербальной подгруппы $\varphi(A * B)$ относительно слова $\varphi$ бесконечна тогда и только тогда, когда $|A| \geqslant 3$ и $|B| \geqslant 2$.

Опираясь на результат А. Х. Ремтулы, В.Г. Бардаковым доказана

Teopema 1 [3]. Пусть $G=A *_{U} B,|A: U| \geqslant 3 u|B: U| \geqslant 2$, причем $U \triangleleft A u U \triangleleft B$, $\varphi$ - собственное слово. Тогда ширина вербальной подгруппы $\varphi(G)$ бесконечна.

Нами будет доказана следующая

Tеорема 2. Пусть $G=A *_{U} B,|A: U| \geqslant 2$ и в $B$ существует такой әлемент $b$, что $U b U \neq U b^{-1} U$. Тогда $G=A *_{U} B$ имеет бесконечную ширину для любого собственного слова $\varphi$.

Прежде чем провести доказательство, введем следующие определения.

Представим всякий элемент $f \neq u, u \in U$, из $G$ в виде

$$
f=g_{1} g_{2} \ldots g_{m},
$$

где $g_{i} \in A$ либо $g_{i} \in B, g_{i} \notin U$ и $g_{i}, g_{i+1}$ - принадлежат разным сомножителям $A, B$.

Элементы $g_{i}$ назовем множителями слова $f$. Пусть $l=v_{1} v_{2} \ldots v_{n}$ - представление (1) элемента $l$. Будем говорить что в произведении $f l$ имеет место слияние, если $f l=g_{1} g_{2} \ldots g_{m-1}\left(g_{m} v_{1}\right) v_{2} \ldots v_{n}$, где $g_{m} v_{1} \in A$ либо $g_{m} v_{1} \in B$, но $g_{m} v_{1} \notin U$.

В произведении $f l$ имеет место сокращение, если $f l=g_{1} \ldots g_{m-1}^{\prime} v_{2} \ldots v_{n}$ и $g_{m-1}^{\prime}=$ $g_{m-1} u, u=g_{m} v_{1}$ и $u \in U$, причем между словами $g_{1} \ldots g_{m-1}^{\prime}, v_{2} \ldots v_{n}$ возможно либо сокращение, либо слияние.

Работа выполнена при финансовой поддержке Российского фонда фундаментальных исследований, грант № 00-01-00767. 
ДокАЗАТЕЛЬСтво. По условию существует элемент $b \in B$ такой, что $U b U \neq U b^{-1} U$. Пусть $f=g_{1} g_{2} \ldots g_{m}$ - представление (1) элемента $f$.

Если $g_{j} \in B$ и $g_{j}=u^{\prime} b^{\varepsilon} u^{\prime \prime}$, где $u^{\prime}, u^{\prime \prime} \in U, \varepsilon= \pm 1$, то заменим $g_{j}$ на $u^{\prime} b^{\varepsilon} u^{\prime \prime}$, причем если $1<j<m$, то $u^{\prime}$ присоединяем к $g_{j-1}$, а $u^{\prime \prime}$ к $g_{j+1}$. Если $j=1$, то $f=u^{\prime} b^{\varepsilon} u^{\prime \prime} g_{2} \ldots g_{m}$ и $u^{\prime \prime}$ присоединим к $g_{2}$, а $u^{\prime}$ оставим. Если $j=m$, то $f=g_{1} g_{2} \ldots g_{m-1} u^{\prime} b^{\varepsilon} u^{\prime \prime}$. В этом случае $u^{\prime}$ присоединяем к $g_{m-1}$, а $u^{\prime \prime}$ оставим.

Такое представление назовем специальной формой элемента $f$. Если $f \in U$, то его специальная форма совпадает с этим элементом. Рассмотрим случай, когда $\varphi-$ собственное некоммутаторное слово. Если случается, что элемент $b$ встречается в специальной форме слова $x$ более двух раз, то отрезок слова $x$ от одного появления $b$ до другого назовем $b$-отрезком длины $2 r-1$, где $2 r-1$ - количество множителей между двумя $b$.

Обозначим $\sigma_{k}(x)$ количество $b$-отрезков длины $2 k-1, k \in \mathbb{N}$, в $x$ и $\sigma_{k}^{*}(x)$ количество $b^{-1}$-отрезков длины $2 k-1$.

Пусть $\varphi\left(x_{1}, x_{2}, \ldots, x_{n}\right)=x_{1}^{r_{1}} \ldots x_{n}^{r_{n}} \varphi^{*}(x)$, где $\varphi^{* x}-$ коммутаторное слово. Пусть $e=\mathrm{HOД}\left(r_{1}, \ldots, r_{n}\right)$; так как $\varphi-$ собственное, то $e \neq 1$.

Определим $\gamma(x)$ как количество $b$-отрезков длины $2 k-1$, для которых $\sigma_{k}(x) \not \equiv \sigma_{k}^{*}(x)$ $(\bmod e)$.

Покажем, что для каждого фиксированного целого $p>1$ функция $\gamma$ ограничена на произведении числа $p$-значений, но неограничена на $\varphi(G)$.

Для любого $x \in G$

$$
\begin{gathered}
\sigma_{k}(x)=\sigma_{k}^{*}\left(x^{-1}\right), \\
\gamma(x)=\gamma\left(x^{-1}\right), \\
\sigma_{k}(x)-\sigma_{k}^{*}(x)+\sigma_{k}\left(x^{-1}\right)-\sigma_{k}^{*}\left(x^{-1}\right)=0 .
\end{gathered}
$$

Введем, далее, следующее обозначение: если $\psi_{k}$ и $v_{k}-$ функции, зависящие от параметра $k$, принимающего целые неотрицательные значения, то запись $\psi_{k}={ }_{l} v_{k}$ означает, что $\psi_{k}=v_{k}$ для всех значений параметра $k$, за исключением не более $l$ значений.

ЛЕмма 1. Для любых двух $x, y \in G$

$$
\sigma_{k}(x y)-\sigma_{k}^{*}(x y)={ }_{l} \sigma_{k}(x)-\sigma_{k}^{*}(x)+\sigma_{k}(y)-\sigma_{k}^{*}(y),
$$

əде $l=9$.

ДокАЗАТЕЛЬСТВо. Если одно из $x, y$ принадлежит $U$ либо $x, y$ принадлежит одному из множителей $A, B$, то доказательство очевидно. Рассмотрим случаи, когда $x$ и $y$ не принадлежат $U$.

СлучАЙ 1 . Пусть $x=g_{1} \ldots g_{m}, y=v_{1} v_{2} \ldots v_{n}$ в специальной форме и последний слог слова $x$ и начальный слог слова $y$ одновременно не лежат ни в $A$, ни в $B$.

Тогда на стыке $x y$ мы не можем выделить элемент $b$. Следовательно, $b$-отрезки $x y$ - это $b$-отрезки $x$ плюс $b$-отрезки $y$ плюс отрезок от последнего $b$ в $x$ до первого $b$ в $y$. Следовательно, $\sigma_{k}(x y)={ }_{1} \sigma_{k}(x)+\sigma_{k}(y)$.

Аналогично,

$$
\sigma_{k}^{*}(x y)={ }_{1} \sigma_{k}^{*}(x)+\sigma_{k}^{*}(y) .
$$

Таким образом,

$$
\sigma_{k}(x y)-\sigma_{k}^{*}(x y)={ }_{2} \sigma_{k}(x)-\sigma_{k}^{*}(x)+\sigma_{k}(y)-\sigma_{k}^{*}(y) .
$$


СлучАй 2. В произведении $x y$ имеет место слияние, т.е. $g_{m} v_{1} \in A$ либо $g_{m} v_{1} \in B$, но $g_{m} v_{1} \notin U$. Если $g_{m} v_{1} \in B$ и $g_{m} v_{1}=u^{\prime} b^{\varepsilon} u^{\prime \prime}\left(u^{\prime}, u^{\prime \prime} \in U, \varepsilon= \pm 1\right)$, то $g_{m} v_{1}$ заменим на $u^{\prime} b^{\varepsilon} u^{\prime \prime}$, присоединяя $u^{\prime} \mathrm{\kappa} g_{m-1}$, а $u^{\prime \prime} \mathrm{\kappa} v_{2}$. Положим $g_{m-1}^{\prime}=g_{m-1} u^{\prime}, t=b^{\varepsilon}$, $v_{2}^{\prime}=u^{\prime \prime} v_{2}$. Если такого преобразования сделать нельзя, то $g_{m-1}^{\prime}=g_{m-1}, t=g_{m} v_{1}$, $v_{2}^{\prime}=v_{2}$. Обозначим $\bar{x}=g_{1} \ldots g_{m-1} t, y^{\prime}=v_{2}^{\prime} \ldots v_{n}$. Теперь к $\bar{x} y^{\prime}$ применим случай 1 . Следовательно, $\sigma_{k}\left(\bar{x} y^{\prime}\right)-\sigma_{k}^{*}\left(\bar{x} y^{\prime}\right)={ }_{2} \sigma_{k}(\bar{x})-\sigma_{k}^{*}(\bar{x})+\sigma_{k}\left(y^{\prime}\right)-\sigma_{k}^{*}\left(y^{\prime}\right)$.

В зависимости от $g_{m}, v_{1}, t$ возможны следующие случаи.

Пусть $v_{1}=u_{2} b, u_{2} \in U$. Тогда $\sigma_{k}(y)={ }_{1} \sigma_{k}\left(y^{\prime}\right), \sigma_{k}^{*}(y)=\sigma_{k}^{*}\left(y^{\prime}\right)$.

1) $g_{m}=b u_{1}, t=b, u_{1} \in U$; тогда $\sigma_{k}(x)=\sigma_{k}(\bar{x}), \sigma_{k}^{*}(x)=\sigma_{k}^{*}(\bar{x})$. Следовательно, $l=3$.

2) $g_{m}=b u_{1}, t=b^{-1}, u_{1} \in U$; тогда $\sigma_{k}(x)={ }_{1} \sigma_{k}(\bar{x}), \sigma_{k}^{*}(x)={ }_{1} \sigma_{k}^{*}(\bar{x})$ и $l=5$.

3) $\sigma_{m}=b u_{1}, t \neq b, b^{-1}, u_{1} \in U$; тогда $\sigma_{k}(x)={ }_{1} \sigma_{k}(\bar{x}), \sigma_{k}^{*}(x)=\sigma_{k}^{*}(\bar{x})$ и $l=4$.

4) $g_{m}=b^{-1} u_{1}, u_{1} \in U, t=b$; тогда $\sigma_{k}(x)={ }_{1} \sigma_{k}(\bar{x}), \sigma_{k}^{*}(x)={ }_{1} \sigma_{k}^{*}(\bar{x})$ и $l=5$.

5) $g_{m}=b^{-1} u_{1}, u_{1} \in U, t=b^{-1}$; тогда $\sigma_{k}(x)=\sigma_{k}(\bar{x}), \sigma_{k}^{*}(x)=\sigma_{k}^{*}(\bar{x})$ и $l=3$.

6) $g_{m}=b^{-1} u_{1}, t \neq b, b^{-1}, u_{1} \in U$; тогда $\sigma_{k}(x)=\sigma_{k}(\bar{x}), \sigma_{k}^{*}(x)={ }_{1} \sigma_{k}^{*}(\bar{x})$ и $l=4$.

7) $\sigma_{m} \neq b^{-1} u_{1}, b u_{1} t=b^{-1}, u_{1} \in U$; тогда $\sigma_{k}(x)=\sigma_{k}(\bar{x}), \sigma_{k}^{*}(x)==_{1} \sigma_{k}^{*}(\bar{x})$ и $l=4$.

8) $g_{m} \neq b^{-1} u_{1}, b u_{1}, t=b, u_{1} \in U$; тогда $\sigma_{k}(x)={ }_{1} \sigma_{k}(\bar{x}), \sigma_{k}^{*}(x)=\sigma_{k}^{*}(\bar{x})$ и $l=4$.

9) $g_{m} \neq b^{-1} u_{1}, b u_{1} t \neq b^{-1}, b, u_{1} \in U$; тогда $\sigma_{k}(x)=\sigma_{k}(\bar{x}), \sigma_{k}^{*}(x)=\sigma_{k}^{*}(\bar{x})$ и $l=3$. Следуюшие случаи 10)-18) получаются, если $v_{1}=u_{2} b^{-1}$. Они рассматриваются аналогично.

Рассмотрим случаи, когда $v_{1} \neq u_{2} b, u_{2} b^{-1}, u_{2} \in U$. Здесь $\sigma_{k}(y)=\sigma_{k}\left(y^{\prime}\right), \sigma_{k}^{*}(y)=$ $\sigma_{k}^{*}\left(y^{\prime}\right)$.

19) $g_{m}=b u_{1}, t=b, u_{1} \in U$; тогда $\sigma_{k}(x)=\sigma_{k}(\bar{x}), \sigma_{k}^{*}(x)=\sigma_{k}^{*}(\bar{x})$ и $l=2$.

20) $g_{m}=b u_{1}, t=b^{-1}, u_{1} \in U$; тогда $\sigma_{k}(x)={ }_{1} \sigma_{k}(\bar{x}), \sigma_{k}^{*}(x)={ }_{1} \sigma_{k}^{*}(\bar{x})$ и $l=4$.

21) $g_{m}=b u_{1}, t \neq b^{-1}, b, u_{1} \in U$; тогда $\sigma_{k}(x)={ }_{1} \sigma_{k}(\bar{x}), \sigma_{k}^{*}(x)=\sigma_{k}^{*}(\bar{x})$ и $l=3$.

22) $g_{m}=b^{-1} u_{1}, t=b, u_{1} \in U$; тогда $\sigma_{k}(x)={ }_{1} \sigma_{k}(\bar{x}), \sigma_{k}^{*}(x)={ }_{1} \sigma_{k}^{*}(\bar{x})$ и $l=4$.

23) $g_{m}=b^{-1} u_{1}, t=b, u_{1} \in U$; тогда $\sigma_{k}(x)={ }_{1} \sigma_{k}(\bar{x}), \sigma_{k}^{*}(x)={ }_{1} \sigma_{k}^{*}(\bar{x})$ и $l=4$.

24) $g_{m} \neq b u_{1}, b^{-1} u_{1}, t=b, u_{1} \in U$; тогда $\sigma_{k}(x)={ }_{1} \sigma_{k}(\bar{x}), \sigma_{k}^{*}(x)=\sigma_{k}^{*}(\bar{x})$ и $l=3$.

25) $g_{m} \neq b u_{1}, b^{-1} u_{1}, t=b^{-1}, u_{1} \in U$; тогда $\sigma_{k}(x)=\sigma_{k}(\bar{x}), \sigma_{k}(x)={ }_{1} \sigma_{k}^{*}(\bar{x})$ и $l=3$.

26) $g_{m} \neq b u_{1}, b^{-1} u_{1}, t \neq b, b^{-1}, u_{1} \in U$; тогда $\sigma_{k}(x)=\sigma_{k}(\bar{x}), \sigma_{k}^{*}(x)=\sigma_{k}^{*}(\bar{x})$ и $l=2$.

27) $g_{m}=b^{-1} u_{1}, t \neq b^{-1}, u_{1} \in U$; тогда $\sigma_{k}(x)=\sigma_{k}(\bar{x}), \sigma_{k}^{*}(x)={ }_{1} \sigma_{k}^{*}(\bar{x})$ и $l=3$.

СлучАЙ 3 . Если на стыке слов $x$ и $y$ происходит сокращение, то получаем элемент $u \in U$, который присоединяем к последнему слогу в $x$, т.е. если $x=g_{1} \ldots g_{m}, y=$ $v_{1} \ldots v_{n}$, то $x y=g_{1} \ldots g_{m-1}^{\prime} v_{2} \ldots v_{n}$, где $g_{m-1}^{\prime}=g_{m-1} u, u \in U$, и т. д. Последний шаг - слияние. Единственное исключение, когда $x y=u$. В этом случае $x=u y^{-1}$ и результат очевиден.

Пусть $x_{1}=g_{1} \ldots g_{i}^{\prime}, g_{i}^{\prime}=g_{i} u, u \in U, y_{1}=v_{m-i+1} \ldots v_{n}$ те части $x$ и $y$, которые не сократились, т. е. между $x_{1}, y_{1}$ происходит слияние.

Пусть $z=g_{i+1} \ldots g_{m}, z^{-1}=v_{1} \ldots v_{m-i}$.

Для $x$ можно записать

$$
\sigma_{k}(x)-\sigma_{k}^{*}(x)={ }_{2} \sigma_{k}\left(x_{1}\right)-\sigma_{k}^{*}\left(x_{1}\right)+\sigma_{k}(z)-\sigma_{k}^{*}(z) .
$$

Аналогично,

$$
\sigma_{k}(y)-\sigma_{k}^{*}(y)={ }_{2} \sigma_{k}\left(y_{1}\right)-\sigma_{k}^{*}\left(y_{1}\right)+\sigma_{k}\left(z^{-1}\right)-\sigma_{k}^{*}\left(z^{-1}\right) .
$$


Используя (4), имеем из (5) и (6)

$$
\sigma_{k}(x)-\sigma_{k}^{*}(x)+\sigma_{k}(y)-\sigma_{k}^{*}(y)={ }_{4} \sigma_{k}\left(x_{1}\right)-\sigma_{k}^{*}\left(x_{1}\right)+\sigma_{k}\left(y_{1}\right)-\sigma_{k}^{*}\left(y_{1}\right) .
$$

Кроме того, $\sigma_{k}(x y)-\sigma_{k}^{*}(x y)=\sigma_{k}\left(x_{1} y_{1}\right)-\sigma_{k}^{*}\left(x_{1} y_{1}\right)$. Используя случай 2 ,

$$
\sigma_{k}\left(x_{1} y_{1}\right)-\sigma_{k}^{*}\left(x_{1} y_{1}\right)={ }_{5} \sigma_{k}\left(x_{1}\right)-\sigma_{k}^{*}\left(x_{1}\right)+\sigma_{k}\left(y_{1}\right)-\sigma_{k}^{*}\left(y_{1}\right) .
$$

Следовательно,

$$
\sigma_{k}(x y)-\sigma_{k}^{*}(x y)={ }_{9} \sigma_{k}(x)-\sigma_{k}^{*}(x)+\sigma_{k}(y)-\sigma_{k}^{*}(y) .
$$

ЛЕмма 2. Для любих двух $x, y \in G$

$$
\gamma(x y) \leqslant \gamma(x)+\gamma(y)+9
$$

Этот результат непосредственно следует из леммы 1.

ЛЕмма 3. Для любого $[x, y] \in G \quad \gamma([x, y]) \leqslant 27$.

ДокАЗАТЕЛЬСТво. Имеем

$$
\begin{gathered}
\sigma_{k}([x, y])-\sigma_{k}^{*}([x, y])={ }_{9} \sigma_{k}\left(x^{-1} y^{-1}\right)-\sigma_{k}^{*}\left(x^{-1} y^{-1}\right)+\sigma_{k}(x y)-\sigma_{k}^{*}(x y) \\
\sigma_{k}\left(x^{-1} y^{-1}\right)-\sigma_{k}^{*}\left(x^{-1} y^{-1}\right)={ }_{9} \sigma_{k}\left(x^{-1}\right)-\sigma_{k}^{*}\left(x^{-1}\right)+\sigma_{k}\left(y^{-1}\right)-\sigma_{k}\left(y^{-1}\right) \\
\sigma_{k}(x y)-\sigma_{k}^{*}(x y)={ }_{9} \sigma_{k}(x)-\sigma_{k}^{*}(x)+\sigma_{k}(y)-\sigma_{k}^{*}(y) .
\end{gathered}
$$

Эти три равенства вместе с (2) дают

$$
\sigma_{k}([x, y])-\sigma_{k}^{*}([x, y])={ }_{27} 0 .
$$

Таким образом, $\gamma([x, y]) \leqslant 27$.

Лемма 4. Для любого $x \in G \quad \gamma\left(x^{e}\right) \leqslant 20$.

ДокАЗАТЕЛЬСТво. Если $x=u, x \in A$ либо $x \in B$, то доказательство леммы очевидно.

Рассмотрим остальные случаи. Пусть для элемента $x^{e}$ имеем $x^{e}=z^{-1} y^{e} z$, где специальная форма $y$ начинается и заканчивается на элементы из разных сомножителей $A, B$.

Если $y \in A$ (либо $y \in B)$, то $\sigma_{k}\left(y^{e}\right)=\sigma_{k}^{*}\left(y^{e}\right)=0$.

В остальных случаях

$$
\sigma_{k}\left(y^{e}\right)={ }_{1} e \sigma_{k}(y), \quad \sigma_{k}^{*}\left(y^{e}\right)={ }_{1} e \sigma_{k}(y) .
$$

Следовательно, $\sigma_{k}\left(y^{e}\right)-\sigma_{k}^{*}\left(y^{e}\right) \equiv_{2} 0(\bmod e)$. Следовательно, из

$$
\sigma_{k}\left(x^{e}\right)-\sigma_{k}^{*}\left(x^{e}\right)={ }_{18} \sigma_{k}\left(y^{e}\right)-\sigma_{k}^{*}\left(y^{e}\right)
$$

имеем $\gamma\left(x^{e}\right) \leqslant 20$.

Очевидно, что для любой группы $G e$-я степень каждого элемента из $G$ есть $\varphi$-значение. 
Положим

$$
z_{j}=(a b)^{e}\left(a b^{2}\right)^{2 e}(a b)^{e}\left(a b^{2}\right)^{3 e}(a b)^{e} \ldots\left(a b^{2}\right)^{j e}(a b)^{e} .
$$

При этом $z_{j} \in \varphi(G)$ для любого $j$. Кроме того, $\gamma\left(z_{j}\right) \geqslant j-1$. И здесь один и только один $b$-отрезок длины $2 e \cdot i+1, i=2,3, \ldots, j$, исключая $b^{-1}$-отрезки этих длин.

Следовательно, $\varphi(G)$ содержит элементы произвольно больших значений $\gamma$.

$\varphi^{*}$ - коммутаторное слово и поэтому записывается как произведение $s$-коммутаторов для некоторого целого $s=s\left(\varphi^{*}\right)$. По леммам 2 и $3 \gamma\left(\varphi^{*}(x)\right) \leqslant 27 s+9(s-1)$. Так как $e$ делит $r_{i}$, то $\gamma\left(x_{i}^{r_{i}}\right) \leqslant 20$ по лемме 4 . Следовательно,

$$
\gamma(\varphi(x)) \leqslant 20 n+9 n+27 s+9(s-1)=36 s+29 n-9,
$$

и если $x$-произведение $m \varphi$-значений, то

$$
\gamma(x) \leqslant(36 s+29 n-9)+9(m-1) .
$$

Если задано $\varphi$, то определены целые $n$ и $s$. Но $\gamma$ может быть сколь угодно большим. Следовательно, мы доказали теорему для собственного некоммутаторного слова $\varphi$.

Покажем, что теорема справедлива и для коммутаторных слов. Как и раньше, пусть специальная форма $x$ есть $g_{1} g_{2} \ldots g_{n}$. Пусть $(x, b)$ указьвает кратность $b$ в $x$, т. е. количество $b$ в специальной форме.

Аналогично, пусть $\left(x, b^{-1}\right)$ - кратность $b^{-1}$ в $x$.

Положим $w(x)=(x, b)-\left(x, b^{-1}\right)$. Тогда

$$
w(x)+w\left(x^{-1}\right)=0 .
$$

Лемма 5. Для любых $x, y$ из $G$

$$
w(x)+w(y)-3 \leqslant w(x y) \leqslant w(x)+w(y)+3 .
$$

ДокАЗАТЕЛЬСТво. Для случая, когда одно из $x, y \in U$ либо $x, y$ принадлежат одному из множителей $A, B$, доказательство очевидно.

Пусть $x=g_{1} \ldots g_{m}, y=v_{1} \ldots v_{n}$ в специальных формах.

СлучАй 1 . В слове $x y$ нет ни слияния, ни сокращения. В этом случае количество $b$ в $x y$ равно количеству $b$ в $x$ плюс количество $b$ в $y$. Следовательно, $w(x y)=w(x)+w(y)$.

СлучАй 2. В этом случае $x y$ на стыке имеет слияние. Здесь объединяется $g_{m}$ с $v_{1}$. Используя доказательство леммы 1 , запишем

$$
\bar{x}=g_{1} \ldots g_{m-1}^{\prime} t, \quad y^{\prime}=v_{2}^{\prime} \ldots v_{n}
$$

$\bar{x} y^{\prime}$ на стыке не имеет ни объединений, ни сокращений. Поэтому применим случай 1 . Таким образом, $w(x y)=w(\bar{x})+w\left(y^{\prime}\right)$.

Так как только один множитель $v_{1}$ убирается при переходе от $y$ к $y^{\prime}$, то, следовательно, имеем

$$
w\left(y^{\prime}\right)-1 \leqslant w(y) \leqslant w\left(y^{\prime}\right)+1 .
$$

Так как при замене $x$ на $\bar{x}$ количество $b$ может уменьшиться не более чем на 2 , то

$$
w(\bar{x})-2 \leqslant w(x) \leqslant w(\bar{x})+2 .
$$


Таким образом,

$$
w(\bar{x})+w\left(y^{\prime}\right)-3 \leqslant w(x)+w(y) \leqslant w(\bar{x})+w\left(y^{\prime}\right)+3
$$

или

$$
w(x y)-3 \leqslant w(x)+w(y) \leqslant w(x y)+3 .
$$

В общем случае произведение $x y$ влечет за собой сокращение и слияние.

Пусть $z=g_{i+1} \ldots g_{m}$, а $z^{-1}=v_{1} \ldots v_{m-i}$ и $g_{i}, v_{m-i+1}$ сливаются. Как и раньше, запишем $\bar{x}=g_{1} \ldots g_{i}^{\prime}, y^{\prime}=v_{m-i+1} \ldots v_{n}$. Тогда $w(x)=w(\bar{x})+w(z)$. Аналогично, $w(y)=w\left(z^{-1}\right)+w\left(y^{\prime}\right)$. Поэтому $w(x)+w(y)=w(\bar{x})+w\left(y^{\prime}\right)$. По случаю 2 $w\left(\bar{x} y^{\prime}\right)-3 \leqslant w(\bar{x})+w\left(y^{\prime}\right) \leqslant w\left(\bar{x} y^{\prime}\right)+3$. Но $w(x y)=w\left(\bar{x} y^{\prime}\right)$. Поэтому $w(x y)-3 \leqslant$ $w(x)+w(y) \leqslant w(x y)+3$.

Лемма 6. Для любых $x, y \in G$

$$
|w(x y)| \leqslant|w(x)|+|w(y)|+3 .
$$

Этот результат непосредственно следует из леммы 6.

Лемма 7. Для любого $[x, y] \in G \quad|w(x, y)| \leqslant 9$.

ДокАЗАТЕЛЬСтво. Результат получается тремя последовательньми применениями леммы 5:

$$
\begin{gathered}
w\left(x^{-1} y^{-1}\right)+w(x y)-3 \leqslant w([x, y]) \leqslant w\left(x^{-1} y^{-1}\right)+w(x y)+3 \\
w\left(x^{-1}\right)+w\left(y^{-1}\right)-3 \leqslant w\left(x^{-1} y^{-1}\right) \leqslant w\left(x^{-1}\right)+w\left(y^{-1}\right)+3 \\
w(x)+w(y)-3 \leqslant w(x y) \leqslant w(x)+w(y)+3
\end{gathered}
$$

Следовательно, $-9 \leqslant w([x, y]) \leqslant 9$.

ЛЕмма 8. G имеет бесконечную ширину для любого собственного коммутаторного слова $\varphi$.

ДокАЗАТЕльство. Так как собственное коммутаторное слово $\varphi$ может быть представленно в виде произведения $s$ коммутаторов для некоторого целого $s=s(\varphi)$, то любой элемент $z$, являющийся произведением $m \varphi$-значений, должен удовлетворять неравенству $|w(z)| \leqslant 12 s m-3$ по леммам 6 и 7. Выберем любой элемент $a \neq u \in A$. Пусть $b$ удовлетворяет условию теоремы 2 . Тогда $b^{2} \neq 1$ и $G$ содержит нетривиальное свободное произведение $G_{1}=\langle a b a b a\rangle *\langle b a b\rangle$, и так как $G_{1}$ не бесконечно определенная группа диэдра, она имеет своей подгруппой свободную группу бесконечного ранга. Таким образом, $\varphi\left(G_{1}\right) \neq 1$.

Беря, если необходимо, сопряженное, считаем, что $\varphi\left(G_{1}\right)$ содержит элемент $1 \neq g=$ $(a b a b a)^{m_{1}}(b a b)^{n_{1}} \ldots(a b a b a)^{m_{r}}(b a b)^{n_{r}}$.

Так как $g$ - коммутаторное слово, то $r$ не меньше 2 .

Если $|w(g)|>0$, то для всякого положительного целого $t\left|w\left(g^{t}\right)\right| \geqslant t$, так как для произведения $g \cdot g$ применим случай 1 леммы 5 . Если $w(g)=0$, то выберем $\gamma$ следующим образом. Если $n_{r} \neq 1$, то выберем $\gamma$, удовлетворяющее условию $n_{r}+\gamma=1$. Если $n_{r}=1$, то выберем $\gamma$, удовлетворяющее условию $\gamma+n_{r}=-1$. Пусть $g_{1}=b^{-\gamma} g b^{\gamma}$ и для $t>1 g_{t}=b^{-\gamma} g_{t-1} g b^{\gamma}=b^{-\gamma t}\left(g b^{\gamma}\right)^{t}$. Очевидно, $g_{t} \in \varphi(G)$. 
В силу выбора $\gamma$, если $n_{r} \neq 1$, то $\left(g b^{\gamma}, b\right)=(g, b)+1$ и $\left(g b^{\gamma}, b^{-1}\right)=\left(g, b^{-1}\right)-1$. Если $n_{r}=1$, то $\left(g b^{\gamma}, b^{-1}\right)=\left(g, b^{-1}\right)+1$ и $\left(g b^{\gamma}, b\right)=(g, b)-1$. Таким образом, $w(g)=0$ и $w\left(g b^{\gamma}\right) \neq 0$.

Левьй конец множителя $g b^{\gamma}$ лежит в $A$, и правый конец лежит в $B$. Следовательно, в произведении $g b^{\gamma} \cdot g b^{\gamma}$ нет слияния. Поэтому применим случай 1 леммы 5 и для любого положительного $t$

$$
\left|w\left(g b^{\gamma}\right)^{t}\right|=t \cdot\left|w\left(g b^{\gamma}\right)\right| \geqslant t .
$$

Нас интересует $|w|$-значение $g_{t}=b^{-\gamma t}\left(g b^{\gamma}\right)^{t}$. Так как $\left|w\left(b^{-\gamma t}\right)\right| \leqslant 1$, по случаю 1 леммы $5 w\left(g_{t}\right)=w\left(\left(g b^{\gamma}\right)^{t}\right)+w\left(b^{-\gamma t}\right)$. Следовательно, $\left|w\left(g_{t}\right)\right| \geqslant t-1$.

Завершая доказательство леммы, мы завершаем и доказательство теоремы 2.

Отметим, что лемма 8 непосредственно следует из предложения 1 работы [4], доказательство которого проведено другими методами.

В теореме 1 рассмотрен вопрос о ширине собственной вербальной подгрупшы в $G=$ $A *_{U} B$, где $|A: U| \geqslant 3$ и $|B: U| \geqslant 2$ и U - нормальная подгруппа как в А, так и в В. Интерес представляет случай, когда $|A: U| \leqslant 2$ и $|B: U| \leqslant 2$. Если при этом U является подгруппой бесконечной ширины относительно собственного слова, то, как показано в [5], $G$ имеет бесконечную ширину. Рассмотрим случай, когда $U-$ подгруппа конечной ширины.

Лемма 9 [6]. Если $A \triangleleft G и \varphi-$ собственное слово, то

$$
\operatorname{wid}(G, \varphi) \leqslant \operatorname{wid}(G / A, \varphi)+\operatorname{wid}(\varphi(G) \cap A, \varphi) .
$$

ПРЕДЛОЖЕНИЕ. Пусть $G=A *_{U} B,|A: U| \leqslant 2 u|B: U| \leqslant 2, U-$ noдәрynna конечной ширины. Тогда ширина собственной вербальной подгруппы $\varphi(G)$ конечна.

ДокАЗАтЕльство. Рассмотрим случай, когда $|A: U|=|B: U|=2$ и, следовательно, $U \triangleleft A$ и $U \triangleleft B$. Из результата [2] следует, что $\operatorname{wid}(G / U, \varphi)<\infty$. Так как $U$ имеет конечную ширину, то $\operatorname{wid}(\varphi(G) \cap U, \varphi)<\infty$. По лемме 9 имеем $\operatorname{wid}(G, \varphi)<\infty$. Остальные случаи очевидны.

Автор благодарит В.Н. Безверхнего за помощь.

\section{СПИСОК ЦИТИРОВАННОЙ ЛИТЕРАТУРЫ}

[1] Мерзляков Ю. И. Рациональные группы. 2-е изд. М.: Наука, 1987.

[2] Rhemtulla A. H. A problem of bounded expressibility in free products // Proc. Cambridge Phil. Soc. 1969. V. 64. № 3. P. 573-584.

[3] Бардаков В. Г. О ширине вербальных подгрупп некоторых свободных конструкций // Алгебра и логика. 1997. Т. 36. № 5. С. 494-517.

[4] Григорчук Р. И. Ограниченные когомологии групповых конструкций // Матем. заметки. 1996. T. 59. № 4. C. 546-550.

[5] Добрынина И. В. К вопросу о шшрине в свободном произведении с объединением // Известия Тульского гос. ун-та. Сер. Матем. Мех. Информатика. 1999. Т. 5. №1. С. 114-115.

[6] Романьков В. А. О ширине вербальных подгрупп разрешимых групп // Алгебра и логика. 1982. T. 21. №1. C. $60-72$. 